\title{
The pancreas in humans with and without diabetes
}

\author{
Yoshifumi Saisho ${ }^{1}$
}

Received: 8 January 2016 / Accepted: 14 January 2016 / Published online: 3 February 2016

(C) Springer-Verlag Berlin Heidelberg 2016

Keywords Diabetes $\cdot$ Humans $\cdot$ Pancreas

\author{
Abbreviations \\ CT Computed tomography \\ PV Pancreas volume \\ RPW Relative pancreas weight
}

To the Editor: We read the paper by Campbell-Thompson et al with great interest [1]. In this study, they used relative pancreas weight (RPW; pancreas weight $[\mathrm{g}] /$ body weight $[\mathrm{kg}]$ ), attempting to normalise the effects of age, ethnicity and body weight. As a result, RPW showed no association with age and was significantly reduced in patients with type 1 diabetes. There was no correlation between RPW and disease duration.

We have previously reported a comprehensive picture of the physiological and pathological changes in pancreas volume (PV) based on computed tomography (CT) scan images of $>2,000$ participants [2-4]. PV was $\sim 10 \mathrm{~cm}^{3}$ in infancy and linearly increased with age in childhood, presumably along with somatic growth. PV then reached a plateau at $\sim 70 \mathrm{~cm}^{3}$ in adulthood (assuming $1 \mathrm{~cm}^{3}=\sim 1 \mathrm{~g}$ ). In adults, PV was positively correlated with BMI. Therefore, it appears reasonable to use RPW to adjust for the effects of age and BMI.

However, it should be noted that PV then started to decline with age from $\sim 60$ years of age. Therefore, caution is needed

Yoshifumi Saisho

ysaisho@keio.jp

1 Department of Internal Medicine, Keio University School of Medicine, 35 Shinanomachi, Shinjuku-ku, Tokyo 160-8582, Japan when RPW is applied to older individuals; in Fig. 1a of their paper, Campbell-Thompson et al presented the relationship between RPW and age only in individuals of age $\leq 60$ years [1].

It should also be noted that using CT scan images we measured parenchymal volume and fat volume of the pancreas separately [2]. We could determine from this that parenchymal and fat components increased proportionally in childhood, while the fat/parenchymal ratio increased with age in adulthood. In adults, the fat/parenchymal ratio also increased with obesity, suggesting that in adults, the fat rather than the parenchymal component is mainly responsible for any increase in PV with age and body weight gain. Therefore, it is important to consider the possibility that the components of the pancreas differ between children and adults even when RPW is the same.

Finally, in the Campbell-Thompson paper, RPW in those with type 2 diabetes was not significantly different from that in nondiabetic controls [1]. We have reported that total PV and parenchymal PV, but not fat volume, were significantly reduced in individuals with type 2 diabetes compared with age-, sex- and BMI-matched controls [2]. This inconsistency between studies may be due to the smaller sample size in the study by Campbell-Thompson et al ( $n=40$ vs 165 with type 2 diabetes). However, in their study there was also a large difference in BMI between people with type 2 diabetes and controls (33.0 vs $22.5 \mathrm{~kg} / \mathrm{m}^{2}$, respectively) [1], suggesting that increased fat in the pancreas due to obesity is likely to have masked the reduced parenchymal pancreas weight in those with type 2 diabetes. Since the pancreas is infiltrated with adipose tissue (i.e. inter- and intra-lobular fat) $[2,5]$, it is difficult to completely remove fat from the resected pancreas.

Nonetheless, an attempt to develop a measure of normalised pancreas mass is of great interest, and we agree that RPW is simple to calculate and will be a new way to compare 
pancreas mass among groups of different age, ethnicity and body weight. The absence of an apparent correlation between RPW and disease duration in both type 1 and type 2 diabetes may reflect the fact that beta cell function/mass is already reduced by $80-90 \%$ at the time of clinical onset $[6,7]$, which is obviously an area of active investigation.

Acknowledgements The author acknowledges P. C. Butler (University of California Los Angeles, CA, USA) for his constructive suggestions and W. Gray (self-employed) for editing the manuscript.

Funding There is no specific funding for this manuscript.

Duality of interest The author declares that there is no duality of interest associated with this manuscript.

Contribution statement YS solely contributed to writing the manuscript, and is the guarantor of this work.

\section{References}

1. Campbell-Thompson ML, Kaddis JS, Wasserfall C et al (2016) The influence of type 1 diabetes on pancreatic weight. Diabetologia 59: 217-221

2. Saisho Y, Butler AE, Meier JJ et al (2007) Pancreas volumes in humans from birth to age one hundred taking into account sex, obesity, and presence of type-2 diabetes. Clin Anat 20:933-942

3. Saisho Y (2014) Pancreas volume with obesity in Asians: comparison with whites. Pancreas 43:657-659

4. Kou K, Saisho Y, Jinzaki M, Itoh H (2014) Relationship between body mass index and pancreas volume in Japanese adults. JOP: J Pancreas 15:626-627

5. Saisho Y, Butler AE, Manesso E, Elashoff D, Rizza RA, Butler PC (2013) $\beta$-cell mass and turnover in humans: effects of obesity and aging. Diabetes Care 36:111-117

6. Atkinson MA, von Herrath M, Powers AC, Clare-Salzler M (2015) Current concepts on the pathogenesis of type 1 diabetes - considerations for attempts to prevent and reverse the disease. Diabetes Care 38:979-988

7. DeFronzo RA, Eldor R, Abdul-Ghani M (2013) Pathophysiologic approach to therapy in patients with newly diagnosed type 2 diabetes. Diabetes Care 36(Suppl 2):S127-S138 